\title{
Bone repair by periodontal ligament stem cell- seeded nanohydroxyapatite-chitosan scaffold
}

\author{
This article was published in the following Dove Press journal: \\ International Journal of Nanomedicine \\ 9 October 2012 \\ Number of times this article has been viewed
}

\section{Shaohua Ge' \\ Ning Zhao' \\ Lu Wang' \\ Meijiao Yu' \\ Hong Liu² \\ Aimei Song' \\ Jing Huang' \\ Guancong Wang ${ }^{2}$ \\ Pishan Yang'}

'Key Laboratory of Oral Biomedicine of Shandong Province, Department of Periodontology, School of Stomatology, ${ }^{2}$ Center of Bio and Micro/Nano Functional Materials, State Key Laboratory of Crystal Materials, Shandong University, Jinan, China
Correspondence: Pishan Yang Key Laboratory of Oral Biomedicine of Shandong Province, Department of Periodontology, School of Stomatology, Shandong University, 44-I Wenhua Road West, Jinan 2500I2, China

$\mathrm{Tel}+8653188382368$

Fax +86 53I 88382923

Email yangps@sdu.edu.cn
Background: A nanohydroxyapatite-coated chitosan scaffold has been developed in recent years, but the effect of this composite scaffold on the viability and differentiation of periodontal ligament stem cells (PDLSCs) and bone repair is still unknown. This study explored the behavior of PDLSCs on a new nanohydroxyapatite-coated genipin-chitosan conjunction scaffold (HGCCS) in vitro as compared with an uncoated genipin-chitosan framework, and evaluated the effect of PDLSC-seeded HGCCS on bone repair in vivo.

Methods: Human PDLSCs were cultured and identified, seeded on a HGCS and on a genipinchitosan framework, and assessed by scanning electron microscopy, confocal laser scanning microscopy, MTT, alkaline phosphatase activity, and quantitative real-time polymerase chain reaction at different time intervals. Moreover, PDLSC-seeded scaffolds were used in a rat calvarial defect model, and new bone formation was assessed by hematoxylin and eosin staining at 12 weeks postoperatively.

Results: PDLSCs were clonogenic and positive for STRO-1. They had the capacity to undergo osteogenic and adipogenic differentiation in vitro. When seeded on HGCCS, PDLSCs exhibited significantly greater viability, alkaline phosphatase activity, and upregulated the bone-related markers, bone sialoprotein, osteopontin, and osteocalcin to a greater extent compared with PDLSCs seeded on the genipin-chitosan framework. The use of PDLSC-seeded HGCCS promoted calvarial bone repair.

Conclusion: This study demonstrates the potential of HGCCS combined with PDLSCs as a promising tool for bone regeneration.

Keywords: periodontal ligament, stem cells, hydroxyapatite, chitosan, scaffold, tissue engineering

\section{Introduction}

Craniofacial bone defects resulting from trauma, pathology, periodontal disease, and congenital abnormalities represent a common and significant clinical problem. An autograft is the gold standard for bone grafting. However, limited availability and donor site morbidity has hindered its use. ${ }^{1}$ Allografts are good alternatives to autografts but are expensive and carry a risk of disease transmission and adverse host immune reactions. These problems have increased the need for synthetic bone graft substitutes. ${ }^{2}$ One of the promising strategies for the development of bone graft substitutes is use of a porous biodegradable material as a scaffold to create the three-dimensional organization needed for appropriate cell interactions, and to serve as vehicles to deliver and retain cells at a specific site. ${ }^{3}$ Recently, chitosan has garnered substantial interest in bone tissue engineering because of its biological properties, ability to be formed into various 
shapes, and capacity to support osteoblast attachment. ${ }^{4-10}$ However, a pure chitosan scaffold lacks the bioactivity to induce hard tissue formation, which limits its application in bone tissue engineering. The biological properties of chitosan for bone regeneration can be improved by combining it with a calcium phosphate, ${ }^{11-15}$ such as hydroxyapatite. ${ }^{11,14}$ Nanohydroxyapatite has attracted considerable interest for bone tissue engineering due to its compositional similarity to native bone. ${ }^{16}$

In addition to having an appropriate scaffold, another key element in bone tissue engineering is osteoblasts or mesenchymal stem cells. Mesenchymal progenitor cells isolated from dental tissues can be efficiently harvested and are capable of committing to the desired phenotype in combination with prefabricated scaffolds and might be used for various regenerative therapies. ${ }^{17-22}$ Since Seo et $\mathrm{al}^{23}$ first isolated stem cells from human periodontal ligament tissue, periodontal ligament stem cells (PDLSCs) have been regarded as good candidates for both cementumperiodontal ligament complexation as well as adjacent bone tissue regeneration. ${ }^{24-26}$ However, PDLSC proliferation and differentiation are impaired in vivo, where hypoxia and the host response threaten the viability of the implanted cells. ${ }^{27}$ The cell delivery system vehicle may be an important regulator of the performance of PDLSCs, and recent studies have indicated that the surface characteristics of scaffolds, including chemical position, roughness, and topography, especially nanotopography, can profoundly affect cell functioning. ${ }^{28-31}$ PDLSCs can in fact colonize and grow connected to different scaffolds and exhibit differentiation and in vivo regenerative capacity. When seeded in a fibrin sponge, PDLSCs produced abundant extracellular matrix which was positively stained by Alizarin red S. ${ }^{32}$ When seeded on a hydroxyapatite extracellular matrix, rat PDLSCs significantly improved calvarial bone repair. ${ }^{27}$ All these findings confirm that PDLSCs combined with a scaffold can be used in bone tissue engineering as well as regenerative treatment of periodontal disease.

In a previous study using a nontoxic crosslinker (genipin) and a nanocrystalline-induced biomimetic mineralization method, we successfully assembled a nanostructured nanohydroxyapatite on a genipin-crosslinked chitosan framework. Unlike other bone tissue scaffolds, the nanohydroxyapatite-coated genipin-chitosan conjugation scaffold (HGCCS) has fluorescent properties that provide effective ways for imaging the scaffold-cell interface, tracing adhesion, migration, and proliferation of cells by confocal laser scanning microscopy. Moreover, the surface biomimetic apatite nanostructure of HGCCS can promote osteogenic differentiation of rat bone marrow-derived mesenchymal stem cells in vitro. ${ }^{33}$ In this present study, we isolated and cultured human PDLSCs and examined the effect of HGCCS on cell morphology, cell viability, and osteogenic differentiation of PDLSCs. Subsequently, a rat calvarial critical size defect model was used to evaluate the potential of PDLSC-seeded HGCCS constructs in vivo for bone tissue regeneration.

\section{Materials and methods}

Preparation of a genipin-crosslinked chitosan framework and a chitosan-nanohydroxyapatite composite framework was performed as previously described. ${ }^{33}$

\section{PDLSCs collection and cultivation}

Written approval for the human periodontal ligament collection performed in this study was obtained from the medical ethics committee at the Medical School, Shandong University (approval number 2010015), and written informed consent was obtained from each participant. The teeth were collected from clinically healthy premolars extracted for orthodontic reasons. Human periodontal ligament tissue was scraped from the middle third of the root surface as previously described. ${ }^{34}$ Briefly, human periodontal ligament tissue was minced into $1 \mathrm{~mm}^{3}$ cubes, and then digested with $3 \mathrm{mg} / \mathrm{mL}$ collagenase I (Invitrogen, Carlsbad, CA) and $4 \mathrm{mg} / \mathrm{mL}$ dispase II (Invitrogen) for 2 hours at $37^{\circ} \mathrm{C}$. The dissociated cell suspension was filtered through a $70 \mu \mathrm{m}$ cell strainer (BD Falcon, BD Biosciences, Bedford, MA). After cell counting, a single-cell suspension was plated at a concentration of 60 cells $/ \mathrm{cm}^{2}$ on nontreated $10 \mathrm{~cm}$ Petri dishes for selection of a single cell-derived colony, and cultured and incubated for 10-14 days in alpha minimal essential medium ( $\alpha$-MEM, Sigma-Aldrich, St Louis, MO) with $10 \%$ fetal calf serum (Hyclone, Logan, UT), 2 mM L-glutamine (Sigma-Aldrich), 100 mM L-ascorbate-2-phosphate (Wako Pure Chemical Industries, Richmond, VA), $1 \mathrm{mM}$ sodium pyruvate (SigmaAldrich), $50 \mathrm{U} / \mathrm{mL}$ penicillin G (JRH Biosciences, Lenexa, $\mathrm{KS}$ ), and $50 \mathrm{mg} / \mathrm{mL}$ streptomycin (JRH Biosciences). Individual colonies were isolated with colony rings and expanded into individual vessels for further cultivation, as described elsewhere. ${ }^{35,36}$

\section{Immunohistochemistry}

Immunocytochemical staining for detecting the mesenchymal stem cell marker, STRO-1, was conducted as previously described. ${ }^{37}$ PDLSCs were subcultured 
into eight-chamber slides until confluent. The cells were fixed with $4 \%$ paraformaldehyde for 15 minutes, and then incubated with $10 \%$ bovine serum albumin (Sigma-Aldrich) for 30 minutes at room temperature, then with the diluted anti-STRO-1 primary antibody (Santa Cruz Biotechnology, Santa Cruz, CA) or mouse-IgG1 as a negative control overnight at $4^{\circ} \mathrm{C}$, and finally with fluorescent-conjugated secondary antibody at $37^{\circ} \mathrm{C}$ in the dark for 30 minutes. Afterwards, the cell nuclei were counterstained with 4',6diamino-2-phenylindole (DAPI, Sigma-Aldrich) in the dark for 5 minutes. The slides were then mounted and observed under a fluorescence microscope.

\section{Multipotent differentiation of single colony-derived PDLSCs}

Osteogenic differentiation was induced as previously described. ${ }^{37}$ PDLSCs were plated at $8 \times 10^{3}$ cells per well in 96-well plates and cultured in $\alpha$-MEM supplemented with $5 \%$ fetal calf serum, $100 \mathrm{mM} \mathrm{L}$-ascorbate-2-phosphate, $1 \mathrm{mM}$ sodium pyruvate, $50 \mu \mathrm{g} / \mathrm{mL}$ streptomycin, $50 \mathrm{U} / \mathrm{mL}$ penicillin G, $2 \mathrm{mM}$ L-glutamine, $0.1 \mu \mathrm{M}$ dexamethasone (Mayne Pharma, Mulgrave, Australia), and $1.8 \mathrm{mM}$ inorganic phosphate $\left(\mathrm{KH}_{2} \mathrm{PO}_{4}\right.$; $\mathrm{BDH}$ Chemicals, Poole, UK) for 4 weeks, with the medium changed twice weekly. Mineral deposit formation was identified by Alizarin Red staining (Sigma-Aldrich).

Adipogenic differentiation was induced as described elsewhere. ${ }^{37}$ PDLSCs were plated in $\alpha$-MEM supplemented with $5 \%$ fetal calf serum, $100 \mathrm{mM} \mathrm{L}$-ascorbate-2-phosphate, $1 \mathrm{mM}$ sodium pyruvate, $50 \mu \mathrm{g} / \mathrm{mL}$ streptomycin, $50 \mathrm{U} / \mathrm{mL}$ penicillin $\mathrm{G}, 2 \mathrm{mM}$ L-glutamine, $0.1 \mu \mathrm{M}$ dexamethasone, and $60 \mu \mathrm{M}$ indomethacin (Sigma-Aldrich), and cultured for 4 weeks, with the medium changed twice weekly. Formation of lipid-laden fat cells was determined by Oil Red O staining (MP Biomedicals, Solon, OH).

\section{PDLSCs cultured on scaffolds}

HGCCS and genipin-chitosan framework (GCF) specimens were cut into $8 \mathrm{~mm} \times 8 \mathrm{~mm} \times 0.6 \mathrm{~mm}$ samples for cell culture and sterilized using $75 \%$ ethanol for 2 hours in a 24-well plate, washed three times with sterile phosphate-buffered solution, transferred into 24-well culture plates, and then immersed in $\alpha$-MEM overnight. Finally, the scaffolds were seeded with $100 \mu \mathrm{L}$ of cell suspension containing $5 \times 10^{4}$ cells. After 3 hours, another $900 \mu \mathrm{L}$ of culture medium was added to each well, and the culture was maintained at $37^{\circ} \mathrm{C}$ in $5 \%$ $\mathrm{CO}_{2}$ at $95 \%$ humidity.

\section{Scanning electron microscopy observation}

The morphology of the PDLSCs on the two scaffolds was examined using a scanning electron microscope (S-750, Hitachi, Tokyo, Japan). Next, $5 \times 10^{4}$ PDLSCs were seeded into each scaffold in 24-well plastic culture plates. Forty-eight hours after initial seeding, the culture medium was discarded, and the cells were gently washed with phosphate-buffered solution three times. Next, the cells on the scaffolds were fixed with $2.5 \%$ glutaraldehyde for 12 hours. After removing the fixative, the scaffolds were carefully washed with phosphatebuffered solution, and fixed in $1 \%$ osmic acid for 1.5 hours. The scaffolds were then subjected to sequential dehydration for 15 minutes with an ethanol series $(50 \%, 70 \%, 80 \%, 90 \%$, and $95 \%$ ). After replacement of isoamyl acetate, the scaffolds were allowed to dry at a conventional critical point, and were then coated with platinum using an ion sputtering coater (IB-5, Eiko, Japan), and observed under a scanning electron microscope to assess cell attachment and morphology.

\section{Confocal laser scanning microscopy observation}

PDLSCs were seeded into each scaffold in 24-well plastic culture plates. Forty-eight hours after initial seeding, the cellcoated samples were fixed with $3.7 \%$ formaldehyde solution for 10 minutes and then extracted with $0.1 \%$ Triton-X100 (Sigma-Aldrich) for 5 minutes, blocked with phosphatebuffered solution containing 1\% bovine serum albumin (Sigma-Aldrich) for 30 minutes, stained with phalloidin conjugated to Alexa Fluor $488^{\circledR}$ (Invitrogen), and examined at excitation wavelengths of $488 \mathrm{~nm}$ and $633 \mathrm{~nm}$ with a confocal laser scanning microscope (CLSM, Leica, Dmirz, Germany).

\section{Cell viability assay}

Cell viability was determined using the tetrazolium salt MTT (3-[4, 5-dimethylthiazol-2-y1]-2, 5-diphenyltetrazolium bromide) assay. Next, $5 \times 10^{4}$ of PDLSCs were seeded into each scaffold in 24-well plastic culture plates and incubated for 1, 3, 7, and 14 days. Specimens were then gently rinsed with phosphate-buffered solution and transferred into new 24-well culture plates. Next, $100 \mu \mathrm{L}$ of MTT solution $(5 \mathrm{mg} / \mathrm{mL})$ was added into each well and incubated at $37^{\circ} \mathrm{C}$ for 4 hours. The medium was then removed and $750 \mu \mathrm{L}$ of dimethylsulfoxide was added. Finally, $150 \mu \mathrm{L}$ of MTT solution was transferred to a 96-well plate. The absorbance value was measured in a multiwell spectrophotometer (BioRad, Hercules, CA) at $490 \mathrm{~nm}$. 


\section{Alkaline phosphatase activity assay}

First, $5 \times 10^{4}$ PDLSCs were seeded into each scaffold in 24-well plastic culture plates and incubated for 3, 7, and 14 days. The specimens were then gently rinsed with phosphate-buffered solution and transferred to new 24-well culture plates. Cells adhered on the scaffolds were lysed using $500 \mu \mathrm{L}$ of $0.1 \%$ Triton-X100 (Sigma-Aldrich) and vibrated for 30 minutes. Finally, $50 \mu \mathrm{L}$ of the supernatant was transferred to a 96-well plate and $50 \mu \mathrm{L}$ of $4 \mathrm{mmol} / \mathrm{L}$ p-nitrophenyl phosphate (Sigma-Aldrich) was added, followed by incubation at $37^{\circ} \mathrm{C}$ for 30 minutes. The absorbance of the reaction solution was measured at $405 \mathrm{~nm}$ using a microplate reader (Bio-Rad). All the data were normalized for total protein content by dividing the amount of alkaline phosphatase in the cells on different scaffolds.

\section{RNA isolation, cDNA synthesis, and quantitative real-time PCR analysis}

After 3, 7, and 14 days, the cell-seeded HGCCS and GCF scaffolds were rinsed twice with sterile phosphate-buffered solution, and the cells on the scaffolds were digested using trypsin and washed from the samples with phosphate-buffered solution. The resulting cell suspension was centrifuged for 3 minutes at $3000 \mathrm{rpm}$. The supernatant was discarded and the cells were lysed using Trizol reagent (Invitrogen), followed by reverse transcription using Oligo dT primers and Superscript III reverse transcriptase according to the manufacturer's instructions (Invitrogen). Real-time polymerase chain reaction (PCR) was performed with $\mathrm{RT}^{2}$ SYBR Green/ROX qPCR Master Mix (SuperArray Bioscience, Frederick, MD). The cycling parameters used were: 15 minutes at $95^{\circ} \mathrm{C}, 40$ cycles [15 seconds at $95^{\circ} \mathrm{C}, 25$ seconds at $60^{\circ} \mathrm{C}, 10$ seconds at $72^{\circ} \mathrm{C}$, and final extension [held for 3 minutes at $72^{\circ} \mathrm{C}$ ]. Primer sets were used for bone sialoprotein, osteopontin, and osteocalcin, and are shown in Table 1. Expression of $\beta$-actin was used as an internal control.

\section{In vivo experimental design}

PDLSCs at a concentration of $1 \times 10^{7} / \mathrm{mL}$ were seeded onto HGCCS and GCF scaffolds, each with $100 \mu \mathrm{L}$ of cell suspension, cultured at $37^{\circ} \mathrm{C}$ and $5 \% \mathrm{CO}_{2}$ for 4 hours, and then $900 \mu \mathrm{L}$ of culture medium was added. After 2 days, the cell-seeded constructs were ready for in vivo transplantation. Eighteen 8-week-old adult male Sprague-Dawley rats (each weighing 180-220 g) were randomly divided into three groups ( $\mathrm{n}=6$ animals each) and treated with HGCCS + PDLSC, GCF + PDLSC, or no cells and no scaffold (negative control group). The rats were anesthetized with $1 \%$ pentobarbital sodium ( $40 \mathrm{~mL} / \mathrm{kg}$ body weight). The surgical area was shaved and scrubbed with sterile gauze soaked in Betadine ${ }^{\circledR}$, and rinsed with sterile water. A cranial skin incision was made and the subcutaneous tissue, musculature, and periosteum were dissected and reflected to expose the calvaria. Full-thickness defects measuring $5 \mathrm{~mm}$ in diameter were created bilaterally in the parietal bones. After copious irrigation with phosphatebuffered solution, the calvarial defects were implanted with PDLSCs seeded on HGCCS or on GCF, so that the scaffolds completely covered and extended $2-3 \mathrm{~mm}$ beyond the defect. For the negative control group, no cells or scaffold were used, and the defects were left empty. The muscles and subcutaneous tissue were sutured in place and the skin was closed. After 12 weeks, the animals were anesthetized and sacrificed. After perfusion fixation with $4 \%$ paraformaldehyde, the calvarial sites with surrounding bone and soft tissue were harvested and fixed in $4 \%$ paraformaldehyde for 24 hours, and then decalcified in $0.5 \mathrm{M}$ ethylenediamine tetra-acetic acid solution for 2 weeks, dehydrated in ethanol, and embedded in paraffin. The specimens were then serially sectioned and stained with hematoxylin and eosin.

\section{Statistical analysis}

The results are presented as the mean \pm standard deviation of 3-5 replicates for each experiment. The statistical significance of the differences between the groups was assessed by one-way analysis of variance. A $P$ value $<0.05$ was considered to be statistically significant.

\section{Results}

\section{Characteristics of PDLSCs}

To identify the PDLSCs, single-cell colonies were generated from human periodontal ligament-derived cells which formed adherent clonogenic cell clusters of fibroblast-like

Table I Primer sequences for osteogenic markers

\begin{tabular}{|c|c|c|c|c|}
\hline Gene & GenBank no & Product size (bp) & Forward primer $5^{\prime}-3^{\prime}$ & Reverse primer $3^{\prime}-5^{\prime}$ \\
\hline$\overline{B S P}$ & NM_004967 & 123 & ATGGCCTGTGCTTTCTCAATG & AGGATAAAAGTGGCATGCTTG \\
\hline OPN & NM_00I040060 & 92 & GCAGACCTGACATCCAGTACC & GATGGCCTTGTATGCACCATTC \\
\hline $\mathrm{OCN}$ & NM_199173 & 255 & ATGAGAGCCCTCACACTCCTCG & GTCAGCCAACTCGTCACAGTCC \\
\hline
\end{tabular}

Abbreviations: BSP, bone sialoprotein; OPN, osteopontin; OCN, osteocalcin. 
cells (Figure 1A). These colony-forming cell populations were termed PDLSCs. The progeny of PDLSCs exhibited typical spindle-shaped fibroblast-like morphology and were positive for the mesenchymal stem cell marker, STRO-1 (Figure 1B). Moreover, after culture in induction medium for 4 weeks, the periodontal ligament single cell-derived colonies formed minerals and lipid vacuoles (Figure 1C and D).

\section{Scanning electron microscopic images of human PDLSCs on scaffolds}

Adhesion of human PDLSCs on the two types of scaffolds was observed by scanning electron microscopy 2 days after cell seeding. As shown in Figure 2A and B, PDLSCs adhered onto the two scaffolds, and the cells and regenerated matrices filled in the voids. Cells adapted very closely to the surface and exhibited flattened morphology. Contact between adjacent cells was also observed in each case.

\section{CLSM images of human PDLSCs on scaffolds}

The morphology and distribution of viable human PDLSCs after 2 days of culture on the scaffolds were observed by CLSM. Actin filaments were stained using Alexa Fluor 488 phalloidin, and could emit green fluorescence when excited by light at a wavelength of $488 \mathrm{~nm}$, and the fluorescent image of the three-dimensional scaffold when excited at a wavelength of $633 \mathrm{~nm}$. Unlike other chitosan-based bone tissue
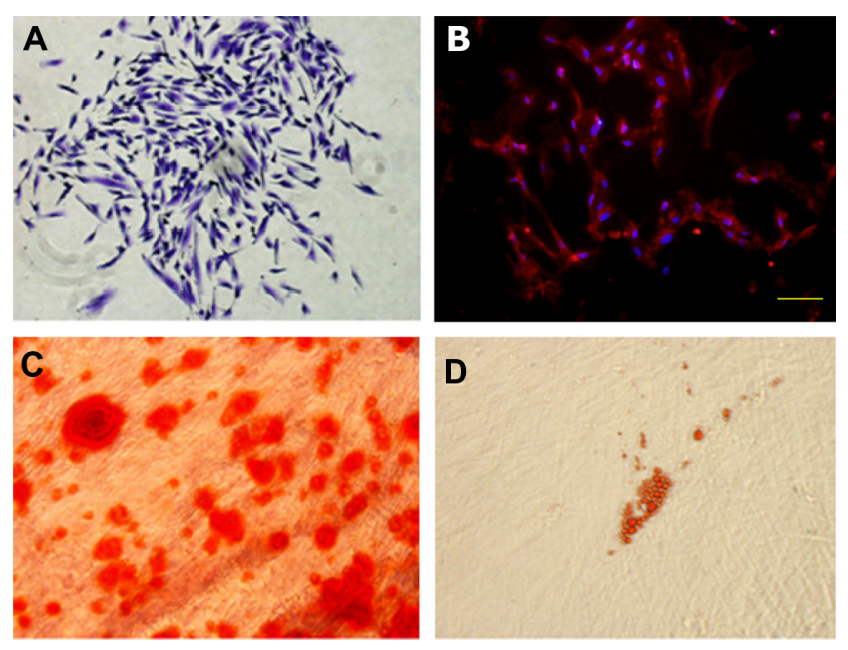

Figure I Characterization of PDLSCs. (A) Cell clusters derived from PDL formed a single colony and were stained with $0.1 \%$ toluidine blue, $\times 100$. (B) Cultured PDLSCs exhibited positive staining for STRO-I $(\times 200)($ STRO-I: red fluorescent signals; nuclei: blue signals). (C) After culture in osteogenic induction media for 4 weeks, cells formed minerals positive for Alizarin Red staining. $\times 400$. (D) After culture in adipogenic induction media for 4 weeks, cells formed lipid vacuoles positive for Oil Red $O$ staining, $\times 200$.

Abbreviations: PDLSCs, periodontal ligament stem cells; PDL, periodontal ligament.
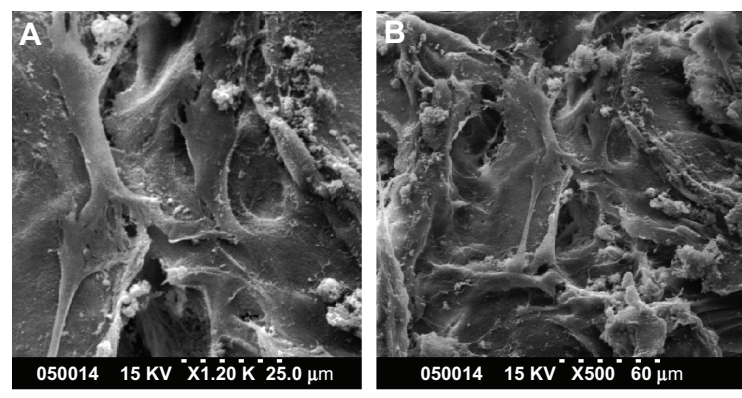

Figure 2 SEM images of PDLSCs on GCF (A) and HGCCS (B).

Notes: PDLSCs adhered on the two scaffolds, the cells and regenerated matrices filled in the voids. Cells adapted very closely to the surface and exhibited flattened morphology.

Abbreviations: SEM, scanning electron microscopy; GCF, genipin-cross-linked chitosan framework; HGCCS, hydroxyapatite coated genipin-chitosan conjugation scaffold.

scaffolds, the fluorescent properties of genipin-crosslinked chitosan show promise for applications in scaffold imaging and tracing, and observation of the interplay between cell biomaterials. Figure 3A and B show the cell morphology and distribution of viable cells on GCF and HGCCS. The cells showed good adhesion with the interconnected channels of three-dimensional porous GCF and HGCCS.

\section{Cell viability}

An MTT assay was used to evaluate the effect of the scaffolds on cell viability. In this study, there was no statistically significant difference between the GCF framework and HGCCS scaffolds after seeding for one day. However, 3,5 , and 7 days after seeding, PDLSCs on HGCCS showed significantly higher viability than those on GCF (Figure 4, $P<0.05)$.

\section{Alkaline phosphatase activity}

Alkaline phosphatase activity is a well defined marker of osteogenesis and is assumed to reflect the degree of differentiation. ${ }^{33}$ In this study, the alkaline phosphatase activity of PDLSCs on GCF and HGCCS was measured for up to 14 days. The results show that the alkaline phosphatase activity of the cells on both scaffolds peaked after 7 days of cultivation but decreased by day 14 (Figure 5). PDLSCs on HGCCS showed higher alkaline phosphatase activity than those on GCF on days 3 and $7(P<0.05)$. On day 14 , there was no significant difference in alkaline phosphatase activity between the two groups.

\section{Real-time PCR analysis of bone-related genes}

In order to explore the differentiating effect of the scaffolds on PDLSCs, expression of osteogenic differentiation-related 

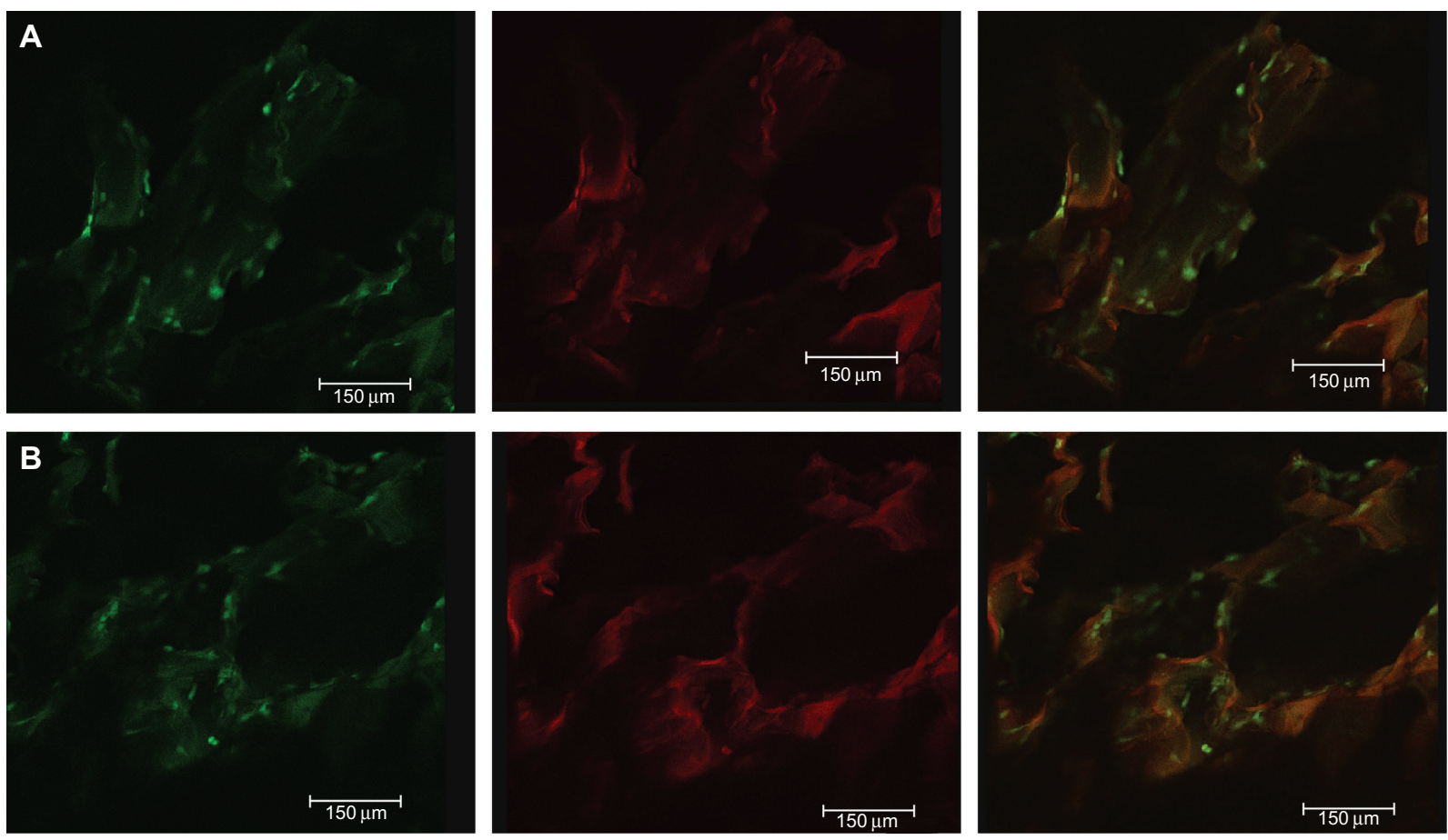

Figure 3 CLSM images of PDLSCs on GCF (A) and HGCCS (B) after 2 days culture in vitro. (AI) Green signals represent cells. Actin filament of the cells was stained by Alexa-fluor 488-phalloidin, which could emit green fluorescence when excited by light with a wavelength of $488 \mathrm{~nm}$. (A2) Red signals represent scaffolds, genipin-chitosan conjugation scaffold possesses fluorescent properties which could emit red fluorescence when excited by light with a wavelength of $633 \mathrm{~nm}$ by a confocal laser scanning microscope. (A3) is the merge of (AI and $\mathbf{A} 2)$. It is the same representation for (B).

Abbreviations: CLSM, confocal laser scanning microscopy; PDLSCs, periodontal ligament stem cells; GCF, genipin-cross-linked chitosan framework; HGCCS, hydroxyapatite coated genipin-chitosan conjugation scaffold.

genes was analyzed by real-time PCR. Bone sialoprotein and osteopontin showed significant upregulation on HGCCS than on pure GCF at day $7(P<0.05$, Figure 6), followed by decreased expression on day 14 , with no significant difference at this time point. Osteocalcin was continuously upregulated on HGCCS, and peaked on day 14. Osteocalcin expression was significantly greater on HGCCS than on GCF on both day 7 and day $14(P<0.05)$.

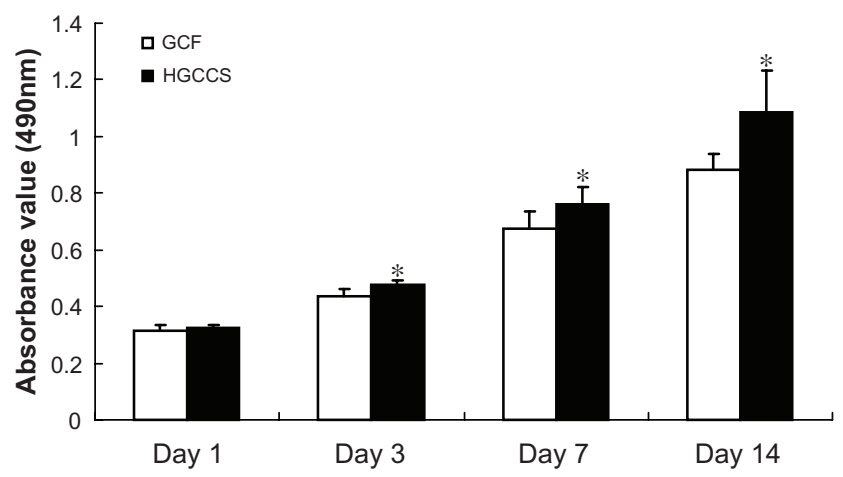

Figure 4 Metabolic activity (MTT assay) of PDLSCs seeded on GCF and HGCCS. Note: Data represents means \pm standard deviation $(n=5$, five replicates per timepoint for each experimental condition). ${ }^{*} P<0.05$, GCF versus HGCCS.

Abbreviations: PDLSCs, periodontal ligament stem cells; GCF, genipin-cross-linked chitosan framework; HGCCS, hydroxyapatite coated genipin-chitosan conjugation scaffold.

\section{In vivo implantation results}

Hematoxylin and eosin staining revealed that the bone defects were filled with newly formed dense tissue in the HGCCS + PDLSC group. As shown in Figure 7A, nondegradable scaffolds were scattered on the surface layer of fibrous connective tissue in the HGCCS + PDLSC group, and new bone formed bony islands along the edges of the defects in the underlying layer. At higher magnification, plenty of

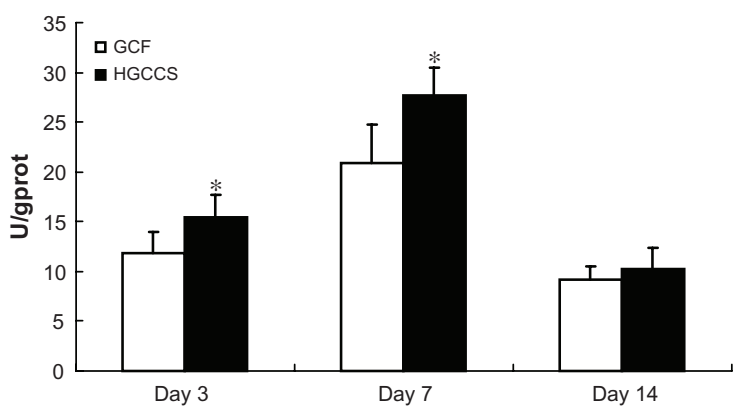

Figure 5 ALP activity of PDLSCs seeded on GCF and HGCCS.

Notes: Data were normalized for total protein content to account for the effect of different number of cells on each scaffold. Data represent means \pm standard deviation ( $n=5$, five replicates per time-point for each experimental condition). $* P<0.05$, GCF versus HGCCS.

Abbreviations: ALP, alkaline phosphatase; PDLSCs, periodontal ligament stem cells; GCF, genipin-cross-linked chitosan framework; HGCCS, hydroxyapatite coated genipinchitosan conjugation scaffold; $U$, unit; GPROT, gram protein. 

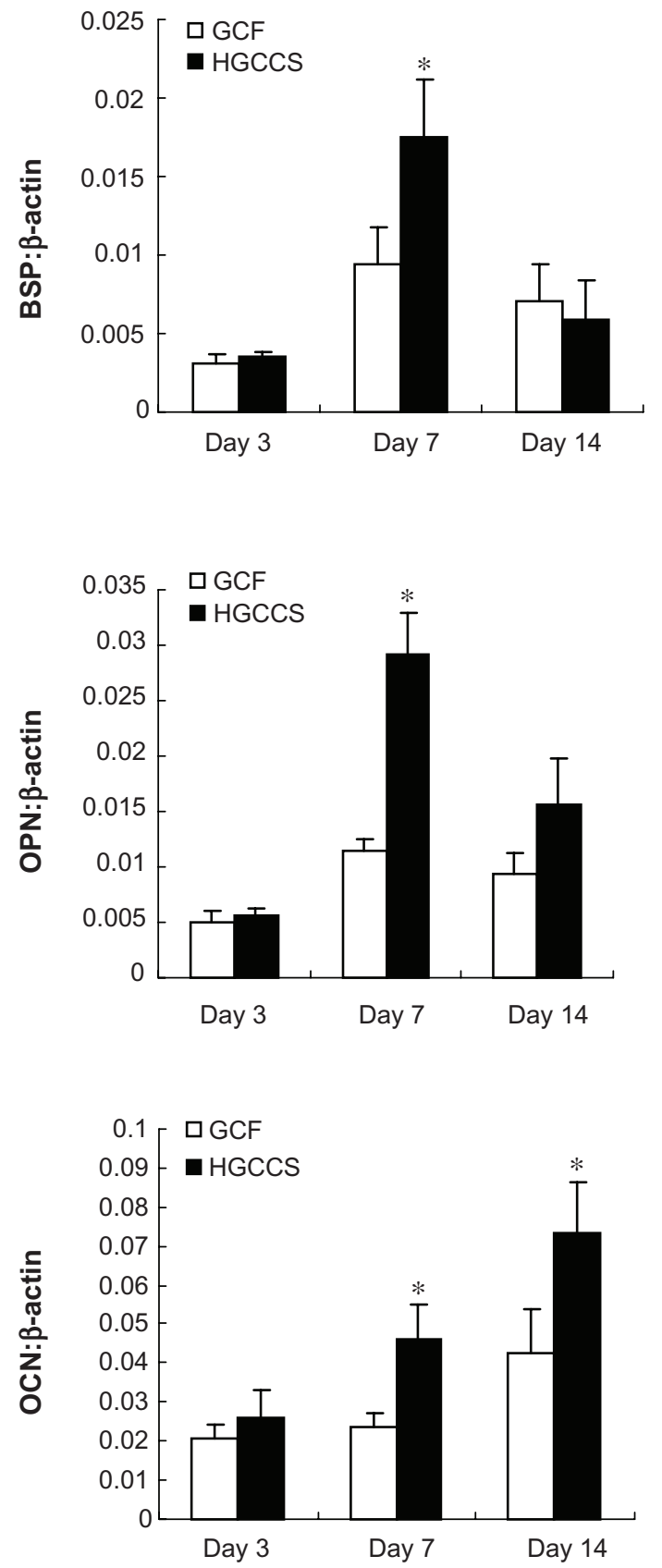

Figure 6 Real-time PCR analysis of BSP, OPN and OCN mRNA expression in PDLSCs on two scaffolds.

Notes: Gene expression is normalized to housekeeping gene $\beta$-actin expression. PDLSCs were cultivated for 3, 7 and 14 days on GCF and HGCCS. Data represent means \pm standard deviation $(n=3)$. $* P<0.05$, GCF versus HGCCS.

Abbreviations: PCR, polymerase chain reaction; BSP, bone sialoprotein; OPN, osteopontin; OCN, osteocalcin; PDLSCs, periodontal ligament stem cells; GCF, genipin-cross-linked chitosan framework; HGCCS, hydroxyapatite coated genipin-chitosan conjugation scaffold.

bone lacunae and osteoblasts were located inside the newly formed bone tissues. Moreover, infiltration of blood vessels was observed throughout the defects (Figure 7D). In the GCF + PDLSC group, new bone formed on the bottom of the scaffold, but the volume of bone seemed lower than in the HGCCS + PDLSC group (Figure 7B and E). The empty defect was used as a negative control to validate the absence of new bone formation in this critically sized bone defect model. The empty defect did not contain any new bone formation as determined by hematoxylin and eosin staining, and contained only a thin layer of fibrous tissue (Figure 7C).

\section{Discussion}

Recent studies have demonstrated that material properties such as surface topography, ${ }^{38}$ matrix stiffness, ${ }^{39}$ hydrophobicity, ${ }^{40}$ and surface chemistry ${ }^{41}$ play an important role in directing the osteogenic differentiation of stem cells. Biomimetic mineralization approaches of assembling a nanostructured apatite layer throughout a three-dimensional porous bone tissue engineering scaffold, ie, biodegradable polymernanohydroxyapatite composites, have provided an effective tool for controlling surface chemistry and geometry within a large and complex structure, and may promote stem cells to differentiate towards osteoblasts. In the current study, we aimed to investigate the effects of a surface biomimetic apatite nanonetwork structure of HGCCS on PDLSC viability and osteogenic differentiation, as well as on bone regeneration capacity in vivo. These characteristics were compared with those of PDLSCs cultured on GCF with a smooth surface.

According to our previous study, HGCCS was constructed using a nanocrystal-induced biomimetic mineralization method. The surface characteristics of HGCCS and GCF were different. The surface of the channel of GCF created by genipin-crosslinked chitosan is clean and smooth. The diameter of the channel is about $150 \mu \mathrm{m}$ and the thickness of the channel wall is about $5 \mu \mathrm{m}$. However, the channel surface of HGCCS is completely covered with a uniform and continuous three-dimensional interconnected nanohydroxyapatite nanonetwork with an approximately $150 \mathrm{~nm}$ pore diameter and a wall thickness of about $20 \mathrm{~nm} \cdot{ }^{33}$ The different surface structures of the two types of scaffold caused a significant difference in the morphology of rat bone marrow-derived mesenchymal stem cells cultured on GCF and HGCCS. On GCF, the vast majority of rat bone marrow-derived mesenchymal stem cells displayed a fibroblast-like shape, while the shape of bone marrow-derived mesenchymal stem cells on the surface of the interconnected nanohydroxyapatite network of HGCCS was polygonal and looked like an osteoblast phenotype. ${ }^{33}$ In the present study, PDLSCs were successfully isolated and cultured using a single-cell-derived colony selection method..$^{35,36}$ They were clonogenic, showed a fibroblast-like morphology, and had a phenotype typical of 

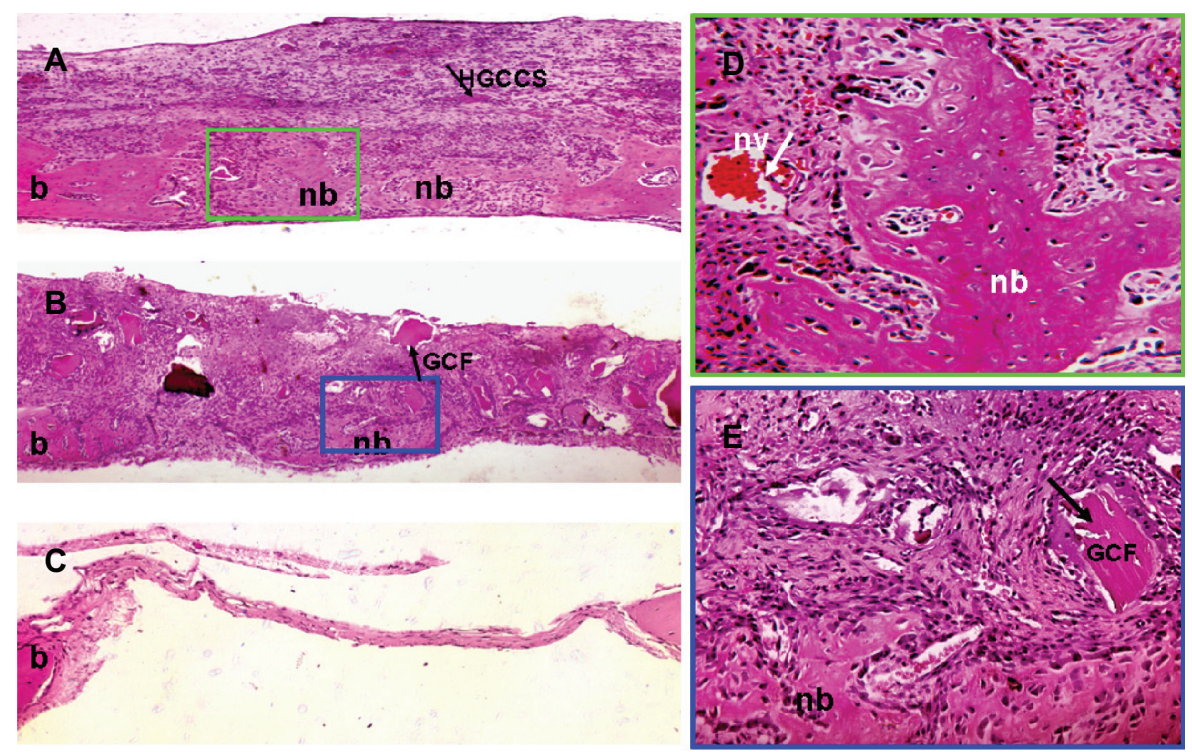

Figure 7 Bone repair capacity of HGCCS + PDLSCs and GCF + PDLSCs. (A) In HGCCS + PDLSCs group, newly formed bone and dense connective tissue covered the defect and there were little sheets of undegradable scaffolds in the upper layer $(\times 100)$. (B) In GCF + PDLSCs group, fairly lower volume of new bone formed on the bottom of the scaffold as compared to HGCCS + PDLSCs group, and undegradable scaffolds could be seen $(\times 100)$. (C) The negative control defect was only filled with thin connective tissue, without bone formation $(\times 100)$. (D) Figure $A$ at higher magnification, a lot of osteoblasts surrounded around the newly formed bone and blood vessel could be seen $(\times 400)$. (E) Figure $B$ at higher magnification $(\times 400)$.

Abbreviations: HGCCS, hydroxyapatite coated genipin-chitosan conjugation scaffold; GCF, genipin-cross-linked chitosan framework; PDLSCs, periodontal ligament stem cells; nb, new bone; b, original bone; nv, new blood vessel.

mesenchymal stem cells. When seeded on HGCCS and GCF, the PDLSCs were found to attach to the two substrates well when observed by scanning electron microscopy. The cells showed uniform attachment and spreading on the surface after 2 days of cultivation. Moreover, on confocal laser scanning microscopy, the cells and regenerated matrices could be seen to grow and fill in the interconnected channels of the threedimensional porous scaffolds. However, the PDLSCs did not change their morphology and there seemed to be no obvious shape difference between the cells on HGCCS and on GCF. A possible reason for the different shapes adopted by PDLSCs and bone-derived mesenchymal stem cells on HGCCS is the different cultivation time for the scaffolds (2 days versus 7 days). Longer exposure to the nanonetwork might alter the shape of the stem cells, which in turn would change the cellular gene expression profiles and signal differentiation in rat bone-derived mesenchymal stem cells.

Although maintaining their intrinsic spindle shape on the two scaffolds, the PDLSCs displayed different viability, alkaline phosphatase activity, and gene expression levels of bone-related markers when cultured on the different scaffolds. Following adhesion, the cells enter into the proliferative phase to colonize and propagate in the porous scaffolds. During the first day of cultivation, no significant difference was observed between the two groups. PDLSCs exhibited higher viability on HGCCS than on pure GCF at day 3 , and this tendency persisted through to day 14. High coverage of nanohydroxyapatite is useful in promoting osteoblast attachment and proliferation. ${ }^{6}$ Moreover, the cells were seen to show increased viability with decreasing pore size until no cells could fit into the pores. ${ }^{42}$ Addition of nanohydroxyapatite decreases the pore size and might increase the scaffold surface area. Therefore, the small pores in the nanohydroxyapatite nanostructure may favor cell growth.

Alkaline phosphatase levels are indicative of osteoblast activity and the early stage of osteoblast differentiation. ${ }^{43}$ After 3 days, alkaline phosphatase activity in PDLSCs on HGCCS was significantly higher than that in those on GCF. Alkaline phosphatase activity in the two groups peaked after 7 days and a significant difference still existed between the two groups. This result suggests that the nanohydroxyapatitecoated scaffold can promote osteogenic differentiation. Similar outcomes regarding induction of higher alkaline phosphatase activity in cells grown on a nanohydroxyapatite substrate have already been reported by our group and by other researchers. ${ }^{33,44}$ Release of calcium and phosphate ions by partial dissolution of the nanohydroxyapatite has been proposed as the possible cause for enhancement of alkaline phosphatase activity. ${ }^{6}$

Real-time PCR analysis of osteogenic gene expression further indicates that the surface microenvironment of the scaffold affects osteogenic differentiation. In this study, gene 
expression of bone sialoprotein, osteopontin, and osteocalcin was upregulated on HGCCS but not on GCF. Bone sialoprotein and osteopontin showed increased upregulation on HGCCS compared with GCF at day 7. Osteocalcin on the other hand showed significant upregulation on HGCCS at both day 7 and day 14. Bone sialoprotein and osteopontin are noncollagenous matrix proteins involved in nucleation of hydroxyapatite crystals and inhibition of mineralization and regulation of cell-matrix and matrix-matrix adhesion, respectively. ${ }^{45,46}$ Osteocalcin is usually used as a marker for the late stage of osteoblast differentiation. ${ }^{43}$ The higher expression level of bone sialoprotein, osteopontin, and osteocalcin by PDLSCs cultured on HGCCS indicates that HGCCS has an enhanced ability to support mineralization of PDLSCs compared with GCF. Surface topography may be one factor promoting the osteogenic commitment of PDLSCs. HGCCS has a vastly different surface topography compared with that of GCF (ie, rough versus smooth), ${ }^{33}$ and a rough topography could contribute to the osteogenic differentiation of mesenchymal stem cells. ${ }^{38}$ In addition to the topographic cues, another factor contributing to HGCCS-mediated osteogenic differentiation of PDLSCs could be the local concentrations of $\mathrm{Ca}$ and $\mathrm{P}$ ions released by nanohydroxyapatite. Higher levels of $\mathrm{Ca}$ and $\mathrm{P}$ ions have been demonstrated to promote osteogenesis of progenitor cells. ${ }^{47}$

Another objective of our study was to evaluate whether PDLSC-seeded scaffolds could promote bone repair when implanted in vivo. A negative control group was included to validate the critical size defect. In our current study, a thin layer of fibrous connective tissue covered the defect without new bone formation in the negative control group. When seeded with HGCCS and PDLSCs, newly formed bone and dense connective tissue covered the defect, and many osteoblasts surrounded the newly formed bone. Moreover, blood vessel infiltration could be seen in the bone repair area, whereas a lower volume of newly formed bone could be seen on the bottom of the scaffold with GCF + PDLSC. In vivo transplantation results demonstrate that the scaffolds had good biocompatibility and supported new bone formation.

Overall, the finding that HGCCS supported more bone formation in vivo is consistent with our in vitro results. Future studies with HGCCS could include exploring the exact mechanisms by which nanostructured apatite induces osteogenic commitment and examining the scaffolds over a longer period of time to evaluate bone growth and scaffold conversion, to enrich our understanding of HGCCS and set up foundations for utilization of HGCCS in clinical practice.

\section{Conclusion}

PDLSCs were successfully isolated and cultured, were found to be clonogenic, and formed minerals and lipid droplets after induction. HGCCS upregulated cell viability and alkaline phosphatase activity. Moreover, HGCCS induced mRNA expression of osteogenic differentiation markers for bone sialoprotein, osteopontin, and osteocalcin. In addition, HGCCS promoted bone repair in vivo when implanted with PDLSCs in a critical size defect model. This study demonstrates that PDLSC-seeded HGCCS constructs are promising for bone tissue repair.

\section{Acknowledgment}

This research was supported by the National Natural Science Foundation of China (81100756, NSFDYS 5092525), Independent Innovation Foundation of Shandong University (2012TS097), Science and Technology Program of Shandong Province (2010GSF10248), and Shandong Province Natural Science Foundation (ZR2009CM118).

\section{Disclosure}

The authors report no conflicts of interest in this work.

\section{References}

1. Giannoudis PV, Dinopoulos H, Tsiridis E. Bone substitutes: an update Injury. 2005;36 Suppl 3:20-27.

2. Bi L, Jung S, Day D, et al. Evaluation of bone regeneration, angiogenesis, and hydroxyapatite conversion in critical-sized rat calvarial defects implanted with bioactive glass scaffolds. J Biomed Mater Res A. June 26, 2012. [Epub ahead of print.]

3. Liao F, Chen Y, Li Z, et al. A novel bioactive three-dimensional beta-tricalcium phosphate/chitosan scaffold for periodontal tissue engineering. J Mater Sci Mater Med. 2010;21(2):489-496.

4. Di Martino A, Sittinger M, Risbud MV. Chitosan: a versatile biopolymer for orthopaedic tissue-engineering. Biomaterials. 2005;26(30): 5983-5990.

5. Fakhry A, Schneider GB, Zaharias R, Senel S. Chitosan supports the initial attachment and spreading of osteoblasts preferentially over fibroblasts. Biomaterials. 2004;25(11):2075-2079.

6. Budiraharjo R, Neoh KG, Kang ET. Hydroxyapatite-coated carboxymethyl chitosan scaffolds for promoting osteoblast and stem cell differentiation. $J$ Colloid Interface Sci. 2012;366(1):224-232.

7. Shi C, Zhu Y, Ran X, Wang M, Su Y, Cheng T. Therapeutic potential of chitosan and its derivatives in regenerative medicine. Surg Res. 2006; 133(2):185-192.

8. Suh JK, Matthew HW. Application of chitosan-based polysaccharide biomaterials in cartilage tissue engineering: a review. Biomaterials. 2000;21(24):2589-2598.

9. Ueno H, Mori T, Fujinaga T. Topical formulations and wound healing applications of chitosan. Adv Drug Deliv Rev. 2001;52(2):105-115.

10. Jayasuriya AC, Bhat A. Fabrication and characterization of novel hybrid organic/inorganic microparticles to apply in bone regeneration. J Biomed Mater Res A. 2010;93(4):1280-1288.

11. Chesnutt BM, Yuan Y, Buddington K, Haggard WO, Bumgardner JD Composite chitosan/nano-hydroxyapatite scaffolds induce osteocalcin production by osteoblasts in vitro and support bone formation in vivo. Tissue Eng Part A. 2009;15(9):2571-2579. 
12. Zhang Y, Zhang M. Cell growth and function on calcium phosphate reinforced chitosan scaffolds. J Mater Sci Mater Med. 2004;15(3): 255-260.

13. Sendemir-Urkmez A, Jamison RD. The addition of biphasic calcium phosphate to porous chitosan scaffolds enhances bone tissue development in vitro. J Biomed Mater Res A. 2007;81(3):624-633.

14. Thein-Han WW, Misra RD. Biomimetic chitosan-nanohydroxyapatite composite scaffolds for bone tissue engineering. Acta Biomater. 2009; 5(4):1182-1197.

15. Zhang Y, Zhang M. Calcium phosphate/chitosan composite scaffolds for controlled in vitro antibiotic drug release. J Biomed Mater Res. 2002;62(3):378-386.

16. Ogata K, Imazato S, Ehara A, et al. Comparison of osteoblast responses to hydroxyapatite and hydroxyapatite/soluble calcium phosphate composites. J Biomed Mater Res A. 2005;72(2):127-135.

17. Isaka J, Ohazama A, Kobayashi M, et al. Participation of periodontal ligament cells with regeneration of alveolar bone. J Periodontol. 2001; 72(3):314-323.

18. Gronthos S, Mankani M, Brahim J, Robey PG, Shi S. Postnatal human dental pulp stem cells (DPSCs) in vitro and in vivo. Proc Natl Acad Sci U S A. 2000;97(25):13625-13630.

19. Yamada Y, Nakamura S, Ito K, et al. A feasibility of useful cell-based therapy by bone regeneration with deciduous tooth stem cells, dental pulp stem cells, or bone marrow-derived mesenchymal stem cells for clinical study using tissue engineering technology. Tissue Eng Part A. 2010;16(6):1891-1900.

20. Laino G, Graziano A, d'Aquino R, et al. An approachable human adult stem cell source for hard-tissue engineering. J Cell Physiol. 2006; 206(3):693-701.

21. Chadipiralla K, Yochim JM, Bahuleyan B, et al. Osteogenic differentiation of stem cells derived from human periodontal ligaments and pulp of human exfoliated deciduous teeth. Cell Tissue Res. 2010; 340(2):323-333.

22. Tomar GB, Srivastava RK, Gupta N, et al. Human gingiva-derived mesenchymal stem cells are superior to bone marrow-derived mesenchymal stem cells for cell therapy in regenerative medicine. Biochem Biophys Res Commun. 2010;393(3):377-383.

23. Seo BM, Miura M, Gronthos S, et al. Investigation of multipotent postnatal stem cells from human periodontal ligament. Lancet. 2004; 364(9249):149-155.

24. Hiraga T, Ninomiya T, Hosoya A, Takahashi M, Nakamura H. Formation of bone-like mineralized matrix by periodontal ligament cells in vivo: a morphological study in rats. $J$ Bone Miner Metab. 2009;27(2):149-157.

25. Huang GT, Gronthos S, Shi S. Mesenchymal stem cells derived from dental tissues vs those from other sources: their biology and role in regenerative medicine. J Dent Res. 2009;88(9):792-806.

26. Kato T, Hattori K, Deguchi T, et al. Osteogenic potential of rat stromal cells derived from periodontal ligament. JTissue Eng Regen Med. 2011; 5(10):798-805.

27. Tour G, Wendel M, Moll G, Tcacencu I. Bone repair using periodontal ligament progenitor cell-seeded constructs. J Dent Res. June 26, 2012. [Epub ahead of print.]

28. Stevens MM, George JH. Exploring and engineering the cell surface interface. Science. 2005;310(5751):1135-1138.

29. Dalby MJ, Gadegaard N, Tare R, et al. The control of human mesenchymal cell differentiation using nanoscale symmetry and disorder. Nat Mater. 2007;6(12):997-1003.
30. Bettinger CJ, Zhang Z, Gerecht S, Borenstein JT, Langer R. Enhancement of in vitro capillary tube formation by substrate nanotopography. $A d v$ Mater. 2008;20(1):99-103.

31. Brammer KS, Oh S, Gallagher JO, Jin S. Enhanced cellular mobility guided by TiO2 nanotube surfaces. Nano Lett. 2008;8(3):786-793.

32. Trubiani O, Orsini G, Zini N, et al. Regenerative potential of human periodontal ligament derived stem cells on three-dimensional biomaterials: a morphological report. J Biomed Mater Res A. 2008; 87(4):986-993.

33. Wang $\mathrm{G}$, Zheng $\mathrm{L}$, Zhao $\mathrm{H}$, et al. In vitro assessment of the differentiation potential of bone marrow-derived mesenchymal stem cells on genipin-chitosan conjugation scaffold with surface hydroxyapatite nanostructure for bone tissue engineering. Tissue Eng Part A. 2011;17(9/10):1341-1349.

34. Wada N, Maeda H, Tanabe K, et al. Periodontal ligament cells secrete the factor that inhibits osteoclastic differentiation and function: the factor is osteoprotegerin/osteoclastogenesis inhibitory factor. $J$ Periodontal Res. 2001;36(1):56-63.

35. Simmons PJ, Torok-Storb B. Identification of stromal cell precursors in human bone marrow by a novel monoclonal antibody, STRO-1. Blood. 1991;78(1):55-62.

36. Nagatomo K, Komaki M, Sekiya I, et al. Stem cell properties of human periodontal ligament cells. J Periodont Res. 2006;41(4):303-310.

37. Du L, Yang P, Ge S. Stromal cell-derived factor-1 significantly induces proliferation, migration, and collagen type I expression in a human periodontal ligament stem cell subpopulation. J Periodontol. 2012; 83(3):379-388.

38. Dalby MJ, Gadegaard N, Curtis AS, Oreffo RO. Nanotopographical control of human osteoprogenitor differentiation. Curr Stem Cell Res Ther. 2007;2(2):129-138.

39. Engler AJ, Sen S, Sweeney HL, Discher DE. Matrix elasticity directs stem cell lineage specification. Cell. 2006;126(4):677-689.

40. Ayala R, Zhang C, Yang D, et al. Engineering the cell-material interface for controlling stem cell adhesion, migration, and differentiation. Biomaterials. 2011;32(15):3700-3711.

41. Benoit DS, Schwartz MP, Durney AR, Anseth KS. Small functional groups for controlled differentiation of hydrogel-encapsulated human mesenchymal stem cells. Nat Mater. 2008;7(10):816-823.

42. O'Brien FJ, Harley BA, Yannas IV, Gibson LJ. The effect of pore size on cell adhesion in collagen-GAG scaffolds. Biomaterials. 2005;26(4): 433-441.

43. Christenson RH. Biochemical markers of bone metabolism: an overview. Clin Biochem. 1997;30(8):573-593.

44. ter Brugge PJ, Wolke JG, Jansen JA. Effect of calcium phosphate coating composition and crystallinity on the response of osteogenic cells in vitro. Clin Oral Implants Res. 2003;14(4):472-480.

45. Ganss B, Kim RH, Sodek J. Bone sialoprotein. Crit Rev Oral Biol Med. 1999;10(1):79-98.

46. McKee MD, Nanci A. Osteopontin at mineralized tissue interfaces in bone, teeth, and osseointegrated implants: ultrastructural distribution and implications for mineralized tissue formation, turnover, and repair. Microsc Res Tech. 1996;33(2):141-164.

47. Chai YC, Roberts SJ, Schrooten J, Luyten FP. Probing the osteoinductive effect of calcium phosphate by using an in vitro biomimetic model. Tissue Eng Part A. 2011;17(7-8):1083-1097.
International Journal of Nanomedicine

\section{Publish your work in this journal}

The International Journal of Nanomedicine is an international, peerreviewed journal focusing on the application of nanotechnology in diagnostics, therapeutics, and drug delivery systems throughout the biomedical field. This journal is indexed on PubMed Central, MedLine, CAS, SciSearch ${ }^{\circledR}$, Current Contents ${ }^{\circledR} /$ Clinical Medicine,

\section{Dovepress}

Journal Citation Reports/Science Edition, EMBase, Scopus and the Elsevier Bibliographic databases. The manuscript management system is completely online and includes a very quick and fair peer-review system, which is all easy to use. Visit http://www.dovepress.com/ testimonials.php to read real quotes from published authors. 